\title{
Multiwater-Assisted Proton Transfer Study in Glycinamide using Density Functional Theory
}

\author{
Ping $\mathrm{Li}^{a, b} \quad$ Yuxiang $B \mathrm{u}^{a, b, *}$ \\ ${ }^{a}$ Institute of Theoretical Chemistry, Shandong University, Jinan 250100, P. R. China \\ ${ }^{b}$ Department of Chemistry, Qufu Normal University, Qufu 273165, P. R. China
}

As mentioned in the paper, additional calculations, such as the full geometry optimization at the MP2(FULL)/6-311++G** level of theory and single-point energy calculations employing higher levels and extended basis sets, have been carried out for the un-, mono-, di-, and trihydrated complexes to further validate the reliability of the B3LYP/6-311++G** level of theory adopted here. Furtherly, on the basis of the computational results, comparisons between them and those results obtained at the B3LYP/6-311++G** level of theory have been made, mainly focusing on the geometrical structures and energetic parameters including interaction energies, tautomeric energies, and barrier heights as described below.

\section{Geometrical structures}

The selected geometrical structures under study have been first displayed in Figure S1. Correspondingly, the standard orientations for those complexes obtained at the MP2(FULL) and B3LYP levels have been listed in Table S1 along with their dipole moments, where both levels can give well-consistent results for these dipole moments obviously.

Overall, analyses of the bond lengths, bond angles, and dihedral angles for those structures suggest that both levels can provide consistent results with each other. In more detail, the largest deviations for bond lengths are not more than $0.03 \AA$, especially for those intermolecular H-bonds formed between fragments in those hydrated complexes. Similarly, the largest deviations among bond angles are within $2^{\circ}$ between both levels. On the other hand, for the dihedral angles, relatively larger geometrical differences can be observed between both levels. For example, the calculated deviations for the main skeleton angles $\mathrm{D}(4,1,2,3)$ are -2.67(1.04)[2.12], 0.57(1.48)[1.59], 5.68(3.35)[3.11], and 2.03(0.58)[3.42] for the reactants (transition states) [products] in the un-, mono-, di-, and trihydrated complexes, where the relatively larger differences can be understood 
since the dihedral angle is a flexible variable relative to other parameters. Most importantly, the strengths of the intermolecular H-bonds formed between fragments in hydrated glycinamidic acid complexes are larger than those of H-bonds in hydrated glycinamide complexes at both levels from the viewpoint of the H-bond contact distances, which can be further supported by the calculated interaction energies below. Furtherly, the changes of angle $\mathrm{A}(\mathrm{O} 5 \mathrm{C} 1 \mathrm{~N} 4)$ for reactant and product relative to its corresponding transition state in the studied proton-transfer processes are well consistent with each other at both levels.

Additionally, single-point energy calculations have also been performed to illustrate the structural differences at both levels, i.e., the energy of the B3LYP/6-311++G** level of theory using the optimized structures at the MP2(FULL)/6-311++G** level of theory and vice versa. As shown in Table S2, the smaller energy differences between both levels may provide another evidence for the smaller structural differences between both levels qualitatively.

\section{Interaction energies}

Table S3 summarizes the calculated interaction energies at the MP2(FULL)/6-311++G** level of theory along with those at the B3LYP level for reference.

Obviously, the interaction energies at the MP2(FULL) level are consistent with those at the B3LYP level within $1.0 \mathrm{kcal} / \mathrm{mol}$ before and after considering ZPVE corrections, where the interaction energy at the B3LYP level is slightly smaller than that at the MP2(FULL) level. Moreover, the differences between both levels increase if further inclusions of BSSE corrections, resulting in the smaller interaction energy at the MP2(FULL) level relative to that at the B3LYP level since the BSSE corrections are much larger at the MP2(FULL) level with the increasing of the water molecules as illustrated in Table S3.

Most importantly, consistent with the B3LYP level, the calculated interaction energies produced in the proton-transferred complexes are also larger than those produced in the non-proton-transferred complexes at the MP2(FULL) level, resulting in the reductions of the tautomeric energy in water-assisted tautomeric processes compared with that of direct tautomeric case in the isolated glycinamide.

\section{Tautomeric energies}

Table S4 presents the calculated tautomeric energies at the various levels along with those at 
the B3LYP/6-311++G** level of theory for reference.

Compared with the MP2(FULL) full optimization level, the calculated tautomeric energies at the B3LYP level are overestimated by about $2 \mathrm{kcal} / \mathrm{mol}$ before and after considering ZPVE corrections, where all the ZPVE corrections at both levels increase the tautomeric energies more or less. Similarly, the same conclusions can be true for the comparisons between the B3LYP level and those single-point energy calculations at higher levels with the $6-311++\mathrm{G}^{* *}$ basis set. Furtherly, at the same level of B3LYP, the tautomeric energies have been decreased slightly by about $1 \mathrm{kcal} / \mathrm{mol}$ at the extended basis sets, suggesting the suitability of the $6-311++\mathrm{G}^{* *}$ basis set adopted here.

Despite of those slight differences among the various levels, however, all the calculations suggest that the participations of the water molecules favor the tautomeric process thermodynamically. Moreover, the tautomeric process assisted with two water molecules is more favorable over others since it has the smallest tautomeric energy after considering ZPVE corrections at all levels.

\section{Barrier heights}

As shown in Table S4, the calculated barrier heights at all levels suggest that the direct participations of water molecules drastically reduce the barrier heights compared with those of the intramolecular proton transfer in isolated glycinamide.

Compared with the MP2(FULL) full optimization level, the calculated barrier heights at the B3LYP level are underestimated by about $1 \sim 3 \mathrm{kcal} / \mathrm{mol}$ except for the forward barrier height from isolated glycinamide to glycinamidic acid, where the barrier height is higher about $1.12 \mathrm{kcal} / \mathrm{mol}$ than that at the MP2(FULL) level. Similarly, analyses of the single-point energy calculations at higher levels like CCSD(T,FULL) also suggest that B3LYP level underestimates the barrier heights more or less. For example, for the forward barrier heights in the un- and monohydrated cases, the largest deviations between CCSD(T,FULL) and B3LYP levels are only $1.5 \mathrm{kcal} / \mathrm{mol}$. On the other hand, the energy difference increases to about $5 \mathrm{kcal} / \mathrm{mol}$ for the reverse barrier height in the dihydrated case. Additionally, at the same level of B3LYP, the deviations between the $6-311++\mathrm{G}^{* *}$ basis set and the extended basis sets have reduced to about $1 \mathrm{kcal} / \mathrm{mol}$, implying the slight influence of the basis set extension. Moreover, the single-point energy results obtained at the MP2(FULL) level are well consistent with those at the MP2(FULL) full optimization level 
within $0.5 \mathrm{kcal} / \mathrm{mol}$, reflecting the geometrical similarity at the B3LYP and MP2(FULL) levels once again.

Most importantly, all the calculations suggest that the proton transfer assisted with two water molecules is the most favorable one among the studied cases since it has the smallest barrier heights regardless of the forward and reverse directions. Thus, the catalytic role of the water molecules has been well reflected at the inexpensive but effective B3LYP/6-311++G** level of theory relative to those of higher levels (e.g., MP2(FULL) level for the present systems) since what we most concerns is to evaluate the optimal numbers of the water molecules in the multi-water assisted proton transfer processes.

In summary, the reliability of the B3LYP/6-311++G** level of theory can be verified from the comparisons with those higher levels and single-point energy calculations mentioned above. Thus, from the viewpoint of the compromise between reliability and computational costs, it is an inexpensive but effective method for studying the glycinamide and its relevant hydrated complexes in the present study. Especially, for the studies of the larger systems formed between glycinamide and relevant molecules of biological importance, such as glycinamide dimer and glycinamide-amino acid complexes, the appliance of the B3LYP level will become more predominant since its lower computational cost allows us to calculate the harmonic vibrational frequencies of these complexes, which are required to investigate their thermochemical properties. 


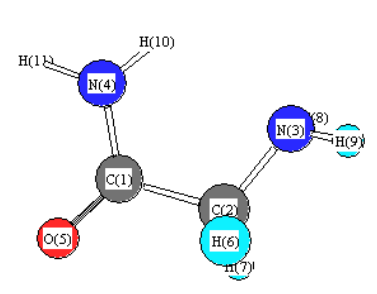

IA

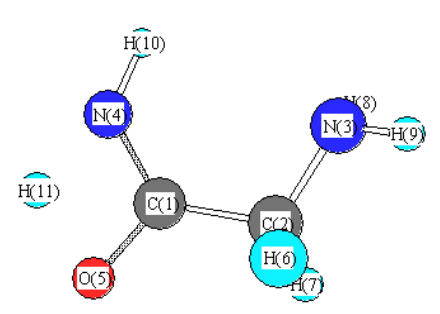

IA*

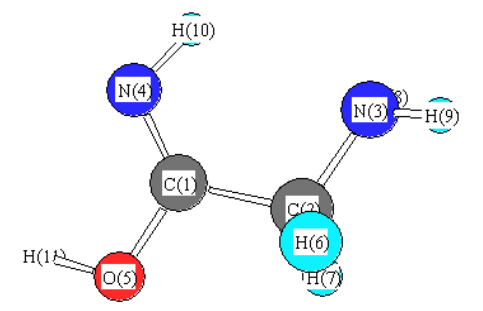

IA'

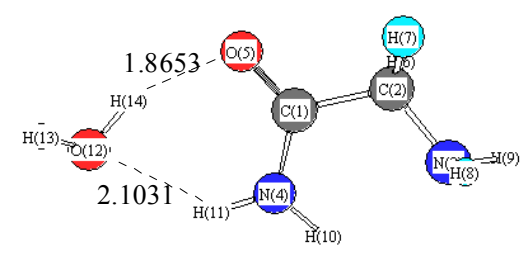

$\mathrm{IA}+1 \mathrm{w}(\mathrm{O} 5 \mathrm{H} 11)$

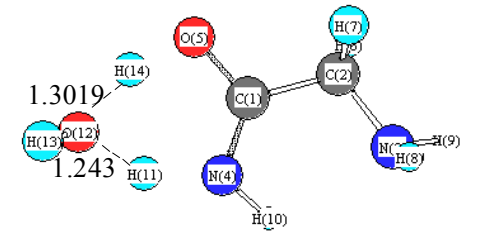

$\mathrm{IA}^{*+1}+\mathrm{w}(\mathrm{O} 5 \mathrm{H} 11)$

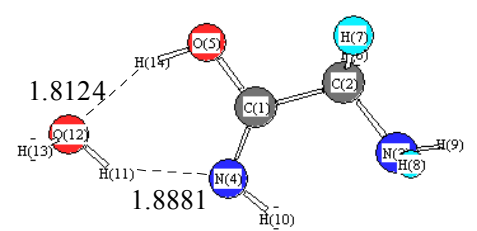

$\mathrm{IA}^{\prime}+1 \mathrm{w}(\mathrm{O} 5 \mathrm{H} 11)$

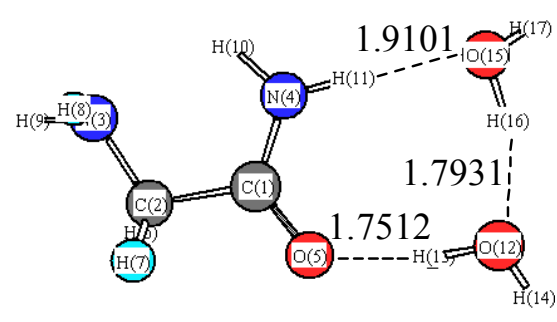

$\mathrm{IA}+2 \mathrm{w}(\mathrm{O} 5 \mathrm{H} 11)$

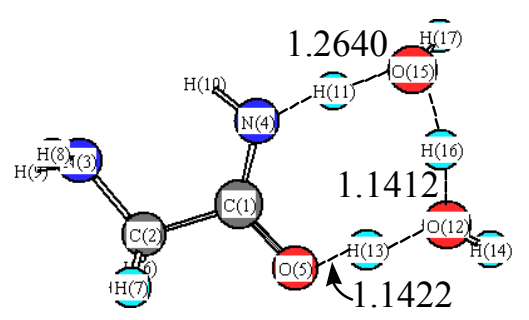

$\mathrm{IA}^{*+}+2 \mathrm{w}(\mathrm{O} 5 \mathrm{H} 11)$

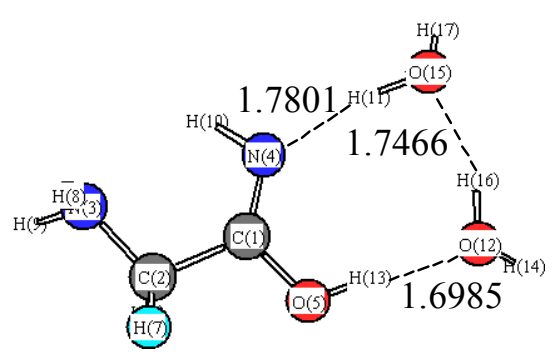

$\mathrm{IA}^{\prime}+2 \mathrm{w}(\mathrm{O} 5 \mathrm{H} 11)$
$\mathrm{IA}^{*+3 \mathrm{w}}(\mathrm{O} 5 \mathrm{H} 11)$

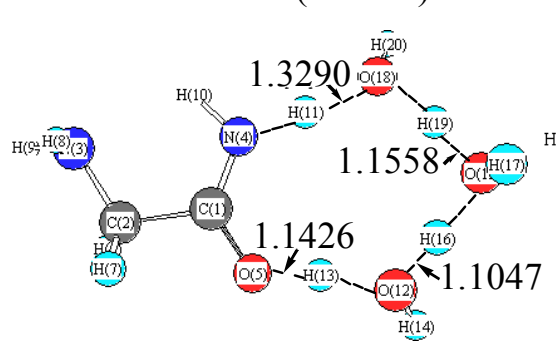

$\mathrm{IA}+3 \mathrm{w}(\mathrm{O} 5 \mathrm{H} 11)$

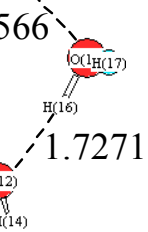$$
\text { (A* } \left.+3 \mathrm{w}^{\circ}(\mathrm{O}) \mathrm{H} 11\right)
$$

(A* $\left.+3 \mathrm{w}^{\circ} \mathrm{OSH}\right)$

Figure S1. Studied complexes for the un-, mono-, di-, and trihydrated glycinamide complexes and their corresponding tautomeric products along with the transition states connecting them, where the intermolecular H-bond distances refer to those obtained at the B3LYP/6-311++G** level of theory. 
Table S1: Standard Orientations for those Studied Complexes in Figure S1 obtained at both the B3LYP/6-311++G** and MP2(FULL)/6-311++G** Levels of Theory. ${ }^{a}$

\begin{tabular}{|c|c|c|c|c|c|c|c|c|}
\hline \multirow{3}{*}{ Proc. } & \multicolumn{4}{|c|}{ B3LYP/6-311++G** } & \multicolumn{4}{|c|}{ MP2(FULL)/6-311++G** } \\
\hline & \multirow{2}{*}{$\begin{array}{l}\text { Atomic } \\
\text { number }\end{array}$} & \multicolumn{3}{|c|}{ Coordinates (in angstroms) } & \multirow{2}{*}{$\begin{array}{l}\text { Atomic } \\
\text { number }\end{array}$} & \multicolumn{3}{|c|}{ Coordinates (in angstroms) } \\
\hline & & $\mathrm{X}$ & $\mathrm{Y}$ & Z & & $\mathrm{X}$ & $\mathrm{Y}$ & Z \\
\hline \multirow{12}{*}{$\mathbf{0}$} & 6 & -0.666945 & -0.088555 & 0.006037 & 6 & -0.662020 & -0.087428 & 0.006363 \\
\hline & 6 & 0.714478 & -0.745268 & 0.117841 & 6 & 0.707925 & -0.753064 & 0.110918 \\
\hline & 7 & 1.828658 & 0.190467 & -0.071117 & 7 & 1.817069 & 0.186780 & -0.056574 \\
\hline & 7 & -0.655292 & 1.267344 & 0.049707 & 7 & -0.630665 & 1.273476 & 0.019515 \\
\hline & 8 & -1.676953 & -0.765372 & -0.092178 & 8 & -1.681889 & -0.755144 & -0.085618 \\
\hline & 1 & 0.226324 & 1.745804 & 0.164833 & 1 & 0.239620 & 1.721736 & 0.266189 \\
\hline & 1 & 0.767384 & -1.159922 & 1.128490 & 1 & 0.702378 & -1.591676 & -0.594137 \\
\hline & 1 & 0.712081 & -1.597185 & -0.570692 & 1 & 0.757217 & -1.179966 & 1.116880 \\
\hline & 1 & 2.087021 & 0.268799 & -1.048694 & 1 & 2.023455 & 0.325973 & -1.039933 \\
\hline & 1 & 2.652948 & -0.104540 & 0.437887 & 1 & 2.658760 & -0.176175 & 0.374685 \\
\hline & 1 & -1.528886 & 1.768287 & 0.032206 & 1 & -1.506582 & 1.762417 & 0.116986 \\
\hline & \multicolumn{4}{|c|}{ Dipole moment: $4.07 \mathrm{D}$} & \multicolumn{4}{|c|}{ Dipole moment: $4.33 \mathrm{D}$} \\
\hline \multirow{12}{*}{$0 *$} & 6 & -0.542714 & -0.062591 & 0.021019 & 6 & -0.539589 & -0.067889 & 0.023472 \\
\hline & 6 & 0.810043 & -0.706079 & 0.097273 & 6 & 0.806780 & -0.715866 & 0.111755 \\
\hline & 7 & 1.884349 & 0.274899 & -0.044247 & 7 & 1.865490 & 0.275575 & -0.052616 \\
\hline & 7 & -0.859573 & 1.201450 & 0.054902 & 7 & -0.836695 & 1.206362 & 0.052033 \\
\hline & 8 & -1.636827 & -0.740826 & -0.089575 & 8 & -1.640061 & -0.732589 & -0.097956 \\
\hline & 1 & -0.182677 & 1.942692 & 0.195305 & 1 & -0.149081 & 1.924399 & 0.253552 \\
\hline & 1 & 0.878476 & -1.172067 & 1.085023 & 1 & 0.881560 & -1.156896 & 1.110143 \\
\hline & 1 & 0.825058 & -1.526708 & -0.632294 & 1 & 0.820429 & -1.544601 & -0.607429 \\
\hline & 1 & 2.051556 & 0.504637 & -1.017805 & 1 & 1.990063 & 0.503956 & -1.032960 \\
\hline & 1 & 2.753011 & -0.072665 & 0.343508 & 1 & 2.748369 & -0.087657 & 0.286776 \\
\hline & 1 & -2.008216 & 0.528291 & -0.041479 & 1 & -1.975562 & 0.550483 & -0.033716 \\
\hline & \multicolumn{4}{|c|}{ Dipole moment: $3.60 \mathrm{D}$} & \multicolumn{4}{|c|}{ Dipole moment: $3.93 \mathrm{D}$} \\
\hline \multirow{12}{*}{ 0' } & 6 & 0.567441 & 0.099754 & -0.020668 & 6 & -0.563395 & 0.096225 & 0.022747 \\
\hline & 6 & -0.709932 & -0.716677 & -0.086287 & 6 & 0.706430 & -0.720345 & 0.105303 \\
\hline & 7 & -1.888304 & 0.134684 & 0.041718 & 7 & 1.873796 & 0.137643 & -0.054640 \\
\hline & 7 & 0.692539 & 1.356515 & -0.046195 & 7 & -0.682197 & 1.362379 & 0.050056 \\
\hline & 8 & 1.652898 & -0.710479 & 0.068599 & 8 & -1.648925 & -0.708850 & -0.080345 \\
\hline & 1 & -0.229794 & 1.784547 & -0.135107 & 1 & 0.256597 & 1.752171 & 0.154590 \\
\hline & 1 & -0.728319 & -1.213412 & -1.061061 & 1 & 0.733227 & -1.183837 & 1.096134 \\
\hline & 1 & -0.638337 & -1.514826 & 0.664097 & 1 & 0.629437 & -1.534966 & -0.625487 \\
\hline & 1 & -2.142577 & 0.284495 & 1.011399 & 1 & 2.100178 & 0.265505 & -1.034501 \\
\hline & 1 & -2.689274 & -0.260305 & -0.433837 & 1 & 2.684670 & -0.268761 & 0.394592 \\
\hline & 1 & 2.430418 & -0.133520 & 0.078782 & 1 & -2.412110 & -0.114745 & -0.078782 \\
\hline & \multicolumn{4}{|c|}{ Dipole moment: 2.74 D } & \multicolumn{4}{|c|}{ Dipole moment: $3.03 \mathrm{D}$} \\
\hline \multirow{4}{*}{1} & 6 & 0.131058 & 0.283394 & -0.017902 & 6 & -0.133420 & 0.290824 & -0.009464 \\
\hline & 6 & 1.622262 & 0.617542 & -0.094651 & 6 & -1.616309 & 0.613259 & 0.131642 \\
\hline & 7 & 2.492239 & -0.548633 & 0.082686 & 7 & -2.472248 & -0.558180 & -0.054237 \\
\hline & 7 & -0.177262 & -1.024525 & -0.065078 & 7 & 0.172328 & -1.022007 & -0.003056 \\
\hline
\end{tabular}




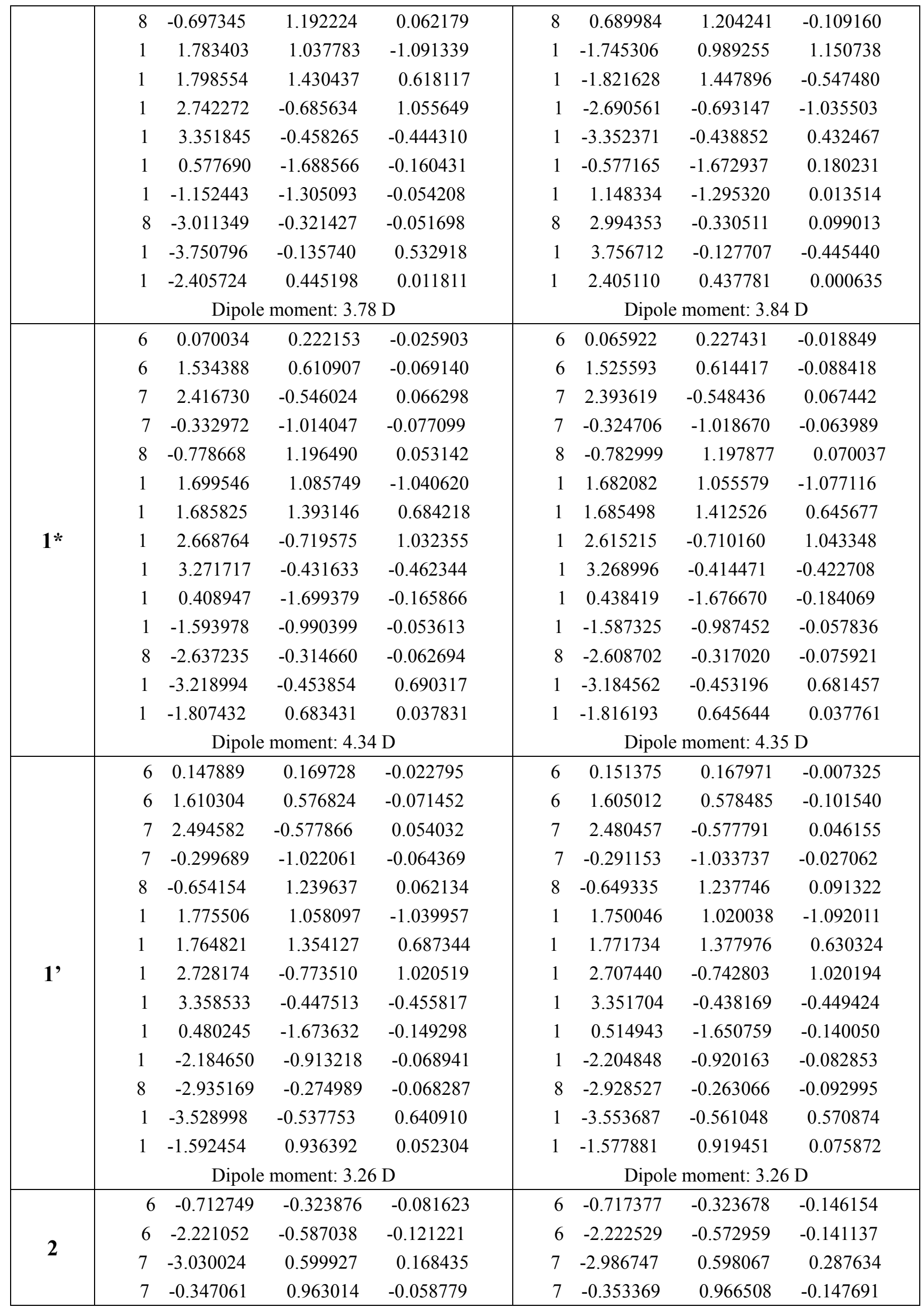




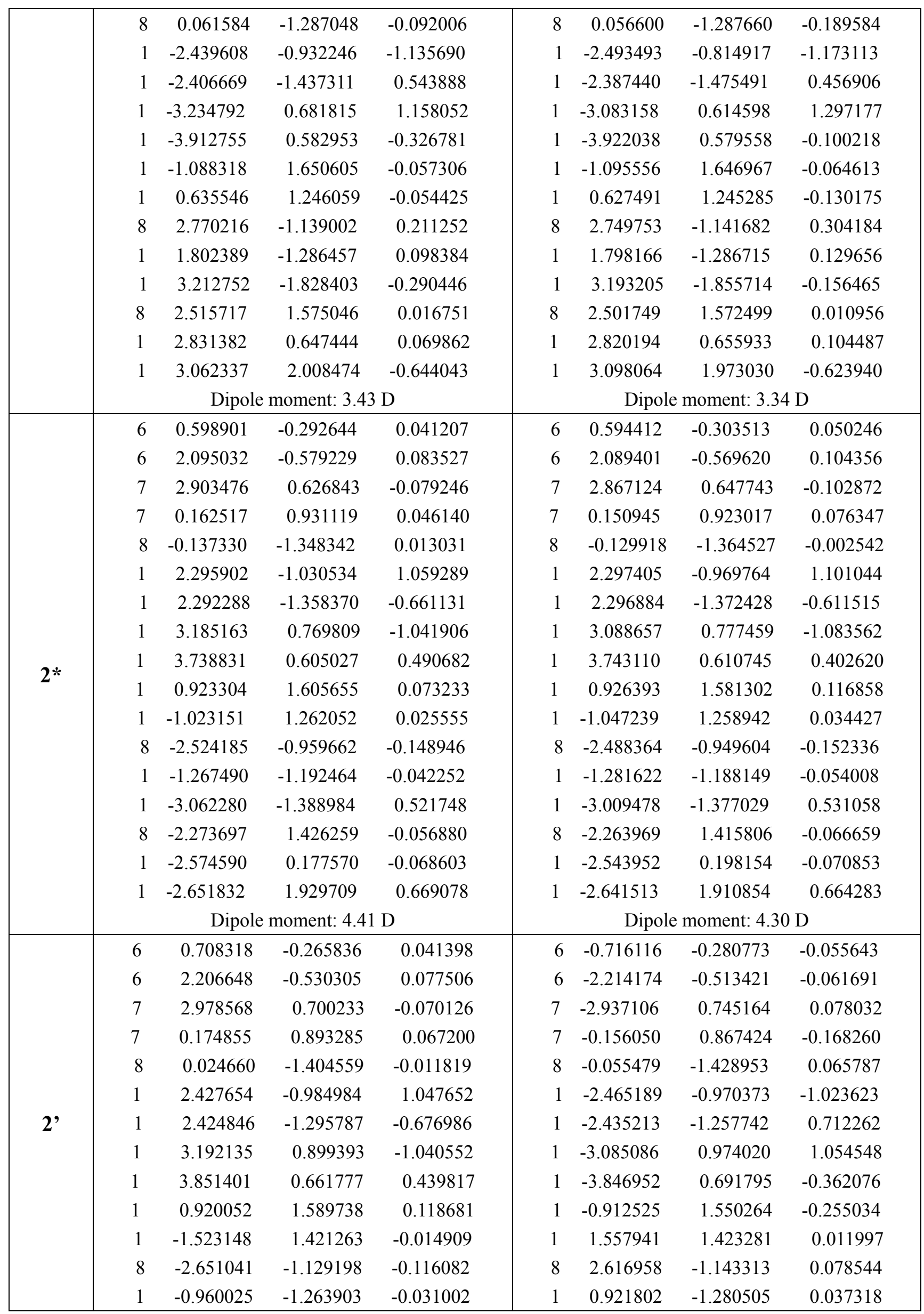




\begin{tabular}{|c|c|c|c|c|c|c|c|c|}
\hline & 1 & -3.228014 & -1.564046 & 0.517794 & 1 & 3.186776 & -1.538943 & -0.583743 \\
\hline & 8 & -2.508826 & 1.551208 & -0.071900 & 8 & 2.528169 & 1.551776 & 0.127642 \\
\hline & 1 & -2.842178 & -0.163239 & -0.068353 & 1 & 2.831415 & -0.189141 & 0.067179 \\
\hline & 1 & -2.744818 & 2.182396 & 0.613321 & 1 & 2.763678 & 2.238301 & -0.499014 \\
\hline & \multicolumn{4}{|c|}{ Dipole moment: $3.27 \mathrm{D}$} & \multicolumn{4}{|c|}{ Dipole moment: $3.38 \mathrm{D}$} \\
\hline \multirow{21}{*}{3} & 6 & -1.278346 & -0.317789 & -0.114389 & 6 & -1.271715 & -0.288858 & -0.208157 \\
\hline & 6 & -2.787538 & -0.578396 & -0.057643 & 6 & -2.770427 & -0.552108 & -0.091646 \\
\hline & 7 & -3.572655 & 0.598735 & 0.323039 & 7 & -3.500593 & 0.567249 & 0.502059 \\
\hline & 7 & -0.909450 & 0.965428 & -0.071154 & 7 & -0.900785 & 0.986727 & -0.048236 \\
\hline & 8 & -0.513829 & -1.283934 & -0.212961 & 8 & -0.513041 & -1.233636 & -0.458891 \\
\hline & 1 & -3.075757 & -0.892456 & -1.064800 & 1 & -3.128191 & -0.708856 & -1.113534 \\
\hline & 1 & -2.929418 & -1.448571 & 0.592376 & 1 & -2.880342 & -1.505119 & 0.437110 \\
\hline & 1 & -3.711608 & 0.649106 & 1.326035 & 1 & -3.518338 & 0.490577 & 1.513147 \\
\hline & 1 & -4.485873 & 0.599173 & -0.113665 & 1 & -4.462868 & 0.577213 & 0.186870 \\
\hline & 1 & -1.651232 & 1.648923 & 0.004035 & 1 & -1.640974 & 1.653106 & 0.123234 \\
\hline & 1 & 0.065667 & 1.269928 & -0.138716 & 1 & 0.064741 & 1.295978 & -0.172402 \\
\hline & 8 & 2.114650 & -1.934560 & 0.015837 & 8 & 2.056145 & -1.954594 & 0.118389 \\
\hline & 1 & 1.169311 & -1.667850 & -0.060703 & 1 & 1.142289 & -1.667820 & -0.080230 \\
\hline & 1 & 2.248249 & -2.623975 & -0.640312 & 1 & 2.210103 & -2.680833 & -0.488657 \\
\hline & 8 & 3.683492 & 0.252381 & 0.302239 & 8 & 3.637858 & 0.235869 & 0.387837 \\
\hline & 1 & 3.187765 & -0.592652 & 0.190671 & 1 & 3.148868 & -0.606540 & 0.292865 \\
\hline & 1 & 4.123146 & 0.193540 & 1.155114 & 1 & 4.066001 & 0.174202 & 1.243556 \\
\hline & 8 & 1.752269 & 2.114569 & -0.226766 & 8 & 1.756133 & 2.096597 & -0.317481 \\
\hline & 1 & 2.494809 & 1.509747 & -0.000730 & 1 & 2.477073 & 1.502206 & -0.031731 \\
\hline & 1 & 2.042309 & 2.595402 & -1.007102 & 1 & 2.087378 & 2.489959 & -1.127007 \\
\hline & \multicolumn{4}{|c|}{ Dipole moment: $3.19 \mathrm{D}$} & \multicolumn{4}{|c|}{ Dipole moment: $3.24 \mathrm{D}$} \\
\hline \multirow{19}{*}{$3 *$} & 6 & -1.134484 & -0.314634 & -0.058553 & 6 & -1.117370 & -0.308308 & -0.102356 \\
\hline & 6 & -2.644441 & -0.539901 & -0.036434 & 6 & -2.624314 & -0.522849 & -0.086419 \\
\hline & 7 & -3.397741 & 0.691188 & 0.186720 & 7 & -3.352644 & 0.685279 & 0.286530 \\
\hline & 7 & -0.669548 & 0.897893 & -0.043293 & 7 & -0.642232 & 0.895092 & 0.057286 \\
\hline & 8 & -0.454078 & -1.400663 & -0.101742 & 8 & -0.442360 & -1.381803 & -0.285865 \\
\hline & 1 & -2.904785 & -0.967043 & -1.008875 & 1 & -2.899307 & -0.827998 & -1.100239 \\
\hline & 1 & -2.837975 & -1.323391 & 0.703962 & 1 & -2.815568 & -1.382118 & 0.564968 \\
\hline & 1 & -3.671410 & 0.803707 & 1.155353 & 1 & -3.599061 & 0.679612 & 1.269177 \\
\hline & 1 & -4.231502 & 0.740056 & -0.383476 & 1 & -4.210385 & 0.776142 & -0.242067 \\
\hline & 1 & -1.420603 & 1.584391 & -0.007271 & 1 & -1.407090 & 1.559470 & 0.166598 \\
\hline & 1 & 0.433983 & 1.305440 & -0.112716 & 1 & 0.474577 & 1.320257 & -0.107087 \\
\hline & 8 & 1.934563 & -1.678994 & 0.027725 & 8 & 1.886804 & -1.655918 & 0.154759 \\
\hline & 1 & 0.687037 & -1.434834 & -0.055886 & 1 & 0.718961 & -1.426910 & -0.083480 \\
\hline & 1 & 2.240366 & -2.264149 & -0.671366 & 1 & 2.224121 & -2.294897 & -0.477862 \\
\hline & 8 & 3.385159 & 0.261883 & 0.183811 & 8 & 3.338189 & 0.251619 & 0.199891 \\
\hline & 1 & 2.635361 & -0.830517 & 0.124457 & 1 & 2.605051 & -0.793572 & 0.205038 \\
\hline & 1 & 3.842108 & 0.386420 & 1.020155 & 1 & 3.784405 & 0.430890 & 1.030449 \\
\hline & 8 & 1.633247 & 1.876904 & -0.148747 & 8 & 1.621910 & 1.851333 & -0.242887 \\
\hline & 1 & 2.601302 & 1.097630 & 0.032153 & 1 & 2.571185 & 1.080705 & -0.012296 \\
\hline
\end{tabular}




\begin{tabular}{|c|c|c|c|c|c|c|c|c|}
\hline & 1 & 1.779564 & 2.432892 & -0.918931 & 1 & 1.730988 & $2.28091 \mathrm{c}$ & -1.094435 \\
\hline & \multicolumn{4}{|c|}{ Dipole moment: $4.70 \mathrm{D}$} & \multicolumn{4}{|c|}{ Dipole moment: $4.04 \mathrm{D}$} \\
\hline \multirow{21}{*}{ 3' } & 6 & -1.292090 & -0.300050 & -0.033075 & 6 & -1.289590 & -0.323610 & -0.044123 \\
\hline & 6 & -2.806546 & -0.465499 & -0.050361 & 6 & -2.795252 & -0.459545 & 0.077021 \\
\hline & 7 & -3.495420 & 0.805015 & 0.157817 & 7 & -3.434877 & 0.849430 & 0.138098 \\
\hline & 7 & -0.690274 & 0.825130 & -0.022687 & 7 & -0.679763 & 0.771990 & -0.313794 \\
\hline & 8 & -0.690125 & -1.482172 & -0.039727 & 8 & -0.693261 & -1.493974 & 0.150218 \\
\hline & 1 & -3.071955 & -0.867855 & -1.032370 & 1 & -3.143017 & -0.990503 & -0.814161 \\
\hline & 1 & -3.062069 & -1.243206 & 0.679219 & 1 & -2.998890 & -1.110654 & 0.935277 \\
\hline & 1 & -3.692297 & 0.974218 & 1.137318 & 1 & -3.492090 & 1.183130 & 1.093767 \\
\hline & 1 & -4.369941 & 0.848624 & -0.348631 & 1 & -4.378567 & 0.809273 & -0.225670 \\
\hline & 1 & -1.402018 & 1.559014 & -0.026668 & 1 & -1.411226 & 1.480014 & -0.422080 \\
\hline & 1 & 0.928298 & 1.550950 & -0.084346 & 1 & 0.942185 & 1.540792 & -0.182672 \\
\hline & 8 & 1.952334 & -1.824132 & -0.018656 & 8 & 1.919265 & -1.798641 & -0.229276 \\
\hline & 1 & 0.303317 & -1.437694 & -0.016049 & 1 & 0.281139 & -1.449591 & -0.016770 \\
\hline & 1 & 2.260675 & -2.439011 & -0.690218 & 1 & 2.222072 & -2.180664 & -1.055259 \\
\hline & 8 & 3.727973 & 0.203531 & 0.170187 & 8 & 3.689455 & 0.174803 & 0.326211 \\
\hline & 1 & 2.651524 & -1.132813 & 0.069429 & 1 & 2.608148 & -1.144405 & 0.009091 \\
\hline & 1 & 4.206741 & 0.368902 & 0.987375 & 1 & 4.116791 & 0.331571 & 1.170170 \\
\hline & 8 & 1.795479 & 2.037840 & -0.140694 & 8 & 1.792787 & 2.033289 & -0.096678 \\
\hline & 1 & 3.100563 & 0.959423 & 0.056895 & 1 & 3.090672 & 0.940110 & 0.195414 \\
\hline & 1 & 1.753537 & 2.561189 & -0.946118 & 1 & 1.808334 & 2.616097 & -0.858428 \\
\hline & \multicolumn{4}{|c|}{ Dipole moment: 2.85 D } & \multicolumn{4}{|c|}{ Dipole moment: $3.01 \mathrm{D}$} \\
\hline
\end{tabular}

${ }^{a}$ The numbers of $0,1,2$, and 3 refer to those of the un-, mono-, di-, and trihydrated cases, respectively.

Table S2: Selected Single-Point Energy Calculations for the Studied Complexes in Figure S1 at the B3LYP/6-311++G** and MP2(FULL)/6-311++G** Levels of Theory. ${ }^{a, b, c}$

\begin{tabular}{c|c|c}
\hline Complexes & $\mathbf{A} / \mathbf{B}-\mathbf{A} / \mathbf{A}$ & $\mathbf{B} / \mathbf{A}-\mathbf{B} / \mathbf{B}$ \\
\hline $\mathbf{0}$ & 0.23 & 0.16 \\
$\mathbf{0}^{*}$ & 0.16 & 0.15 \\
$\mathbf{0}$ & 0.22 & 0.22 \\
$\mathbf{1}$ & 0.23 & 0.21 \\
$\mathbf{1 *}^{*}$ & 0.30 & 0.30 \\
$\mathbf{1}$ & 0.33 & 0.32 \\
$\mathbf{2}$ & 0.32 & 0.28 \\
$\mathbf{2}^{*}$ & 0.36 & 0.35 \\
$\mathbf{2}^{\mathbf{3}}$ & 0.45 & 0.44 \\
$\mathbf{3 *}$ & 0.41 & 0.39 \\
$\mathbf{3}$ & 0.57 & 0.42 \\
\hline
\end{tabular}

${ }^{a}$ The numbers of $0,1,2$, and 3 refer to those of the un-, mono-, di-, and trihydrated cases, respectively.

${ }^{b}$ The A/B refers to the energy calculation of A level (B3LYP/6-311++ $\mathrm{G}^{* *}$ ) using the optimized structure at the B level (MP2(FULL)/6-311++G**) and vice versa.

${ }^{c}$ All the data are in $\mathrm{kcal} / \mathrm{mol}$. 
Table S3: Calculated Interaction Energies, ZPVE Corrections, and BSSE Corrections for the Interactions of Glycinamide and Glycinamidic Acid with Water molecules at the B3LYP/6-311++G** and MP2(FULL)/6-311++G** Levels of Theory. ${ }^{a, b}$

\begin{tabular}{|c|c|c|c|c|}
\hline Complexes & Levels of theory & $\Delta \mathrm{E}_{\text {Interaction }}$ & $\Delta \mathrm{E}_{\mathrm{ZPVE}}$ & $\Delta \mathrm{E}_{\mathrm{BSSE}}$ \\
\hline \multirow{2}{*}{$\mathrm{IA}+1 \mathrm{w}(\mathrm{O} 5 \mathrm{H} 11)$} & B3LYP/6-311++G** & $-9.69(-7.30)[-6.72]$ & 2.39 & 0.58 \\
\hline & MP2(FULL)/6-311++G** & $-10.19(-7.86)[-5.68]$ & 2.33 & 2.18 \\
\hline \multirow{2}{*}{$\mathrm{IA}^{\prime}+1 \mathrm{w}(\mathrm{O} 5 \mathrm{H} 11)$} & B3LYP/6-311++G** & $-11.90(-9.25)[-8.39]$ & 2.65 & 0.86 \\
\hline & MP2(FULL)/6-311++G** & $-12.29(-9.58)[-6.83]$ & 2.71 & 2.75 \\
\hline \multirow{2}{*}{$\mathrm{IA}+2 \mathrm{w}(\mathrm{O} 5 \mathrm{H} 11)$} & B3LYP/6-311++G** & $-20.97(-16.07)[-14.21]$ & 4.90 & 1.86 \\
\hline & MP2(FULL)/6-311++G** & $-21.77(-16.95)[-11.80]$ & 4.82 & 5.15 \\
\hline \multirow{2}{*}{$\mathrm{IA}^{\prime}+2 \mathrm{w}(\mathrm{O} 5 \mathrm{H} 11)$} & B3LYP/6-311++G** & $-23.56(-18.56)[-16.40]$ & 5.00 & 2.16 \\
\hline & MP2(FULL)/6-311++G** & $-24.28(-19.05)[-13.11]$ & 5.22 & 5.95 \\
\hline \multirow{2}{*}{$\mathrm{IA}+3 \mathrm{w}(\mathrm{O} 5 \mathrm{H} 11)$} & B3LYP/6-311++G** & $-31.29(-23.88)[-20.77]$ & 7.41 & 3.12 \\
\hline & MP2(FULL)/6-311++G** & $-32.49(-25.00)[-16.89]$ & 7.49 & 8.11 \\
\hline \multirow{2}{*}{$\mathrm{IA}^{\prime}+3 \mathrm{w}(\mathrm{O} 5 \mathrm{H} 11)$} & B3LYP/6-311++G** & $-33.30(-26.05)[-22.74]$ & 7.34 & 3.30 \\
\hline & MP2(FULL)/6-311++G** & $-34.58(-26.79)[-17.95]$ & 7.79 & 8.83 \\
\hline
\end{tabular}

${ }^{a}$ All the data are in $\mathrm{kcal} / \mathrm{mol}$.

${ }^{b}$ The data in parentheses and brackets refer to the results considering ZPVE corrections and further BSSE corrections, respectively. 
Table S4: Calculated Tautomeric Energies (in $\mathrm{kcal} / \mathrm{mol}$ ) and Barrier Heights (in $\mathrm{kcal} / \mathrm{mol}$ ) for the studied Tautomeric Processes at various Levels of Theory. ${ }^{a, b, c}$

\begin{tabular}{|c|c|c|c|c|}
\hline Proc. & Levels of theory & $\Delta \mathrm{E}$ & $\Delta \mathrm{E}_{\mathrm{f}}^{*}$ & $\Delta \mathrm{E}_{\mathrm{r}}^{*}$ \\
\hline \multirow{8}{*}{$\mathbf{0}$} & $B 3 L Y P / 6-311++G^{* *}$ & 14.32(14.44) & $48.76(45.36)$ & $34.44(30.93)$ \\
\hline & B3LYP/6-311++G(3df,3pd) & $13.67(13.80)$ & $47.78(44.39)$ & $34.10(30.60)$ \\
\hline & B3LYP/AUG-cc-pVTZ & $13.66(13.79)$ & $47.67(44.29)$ & $34.01(30.51)$ \\
\hline & $M P 2(F U L L) / 6-311++G^{* *}$ & $12.56(12.61)$ & $47.73(44.24)$ & $35.17(31.63)$ \\
\hline & MP2(FULL)/6-311++G** & $12.62(12.74)$ & $47.73(44.35)$ & $35.11(31.60)$ \\
\hline & MP3(FULL)/6-311++G** & $10.82(10.95)$ & $51.96(48.57)$ & $41.13(37.63)$ \\
\hline & MP4(SDQ)/6-311++G** & $12.25(12.37)$ & $50.92(47.53)$ & $38.67(35.16)$ \\
\hline & $\operatorname{CCSD}(\mathrm{T}, \mathrm{FULL}) / 6-311++\mathrm{G}^{* *}$ & 11.61(11.73) & $48.71(45.33)$ & $37.10(33.60)$ \\
\hline \multirow{8}{*}{1} & $B 3 L Y P / 6-311++G^{* *}$ & $12.11(12.49)$ & $22.56(19.05)$ & $10.45(6.56)$ \\
\hline & B3LYP/6-311++G(3df,3pd) & $11.76(12.14)$ & 21.99(18.48) & $10.23(6.34)$ \\
\hline & B3LYP/AUG-cc-pVTZ & $11.77(12.15)$ & $21.96(18.45)$ & $10.19(6.30)$ \\
\hline & $M P 2(F U L L) / 6-311++G^{* *}$ & $10.46(10.88)$ & $22.78(19.18)$ & $12.32(8.30)$ \\
\hline & MP2(FULL)/6-311++G** & 10.57(10.95) & $22.87(19.37)$ & $12.30(8.41)$ \\
\hline & MP3(FULL)/6-311++G** & $9.40(9.78)$ & $25.86(22.35)$ & $16.46(12.57)$ \\
\hline & MP4(SDQ)/6-311++G** & 10.73(11.11) & $26.27(22.76)$ & $15.54(11.65)$ \\
\hline & $\operatorname{CCSD}(\mathrm{T}, \mathrm{FULL}) / 6-311++\mathrm{G} * *$ & 9.91(10.29) & $24.10(20.59)$ & $14.18(10.29)$ \\
\hline \multirow{8}{*}{2} & $B 3 L Y P / 6-311++G^{* *}$ & $11.73(11.95)$ & $21.98(16.93)$ & $10.25(4.98)$ \\
\hline & B3LYP/6-311++G(3df,3pd) & $11.25(11.47)$ & $21.36(16.31)$ & $10.11(4.84)$ \\
\hline & B3LYP/AUG-cc-pVTZ & $11.26(11.48)$ & 21.34(16.29) & $10.08(4.81)$ \\
\hline & $M P 2(F U L L) / 6-311++G^{* *}$ & $10.05(10.51)$ & 23.22(17.97) & $13.17(7.46)$ \\
\hline & MP2(FULL)/6-311++G** & $10.21(10.44)$ & $23.29(18.24)$ & $13.08(7.81)$ \\
\hline & MP3(FULL)/6-311++G** & $9.35(9.58)$ & 26.99(21.95) & $17.64(12.37)$ \\
\hline & MP4(SDQ)/6-311++G** & $10.59(10.81)$ & $27.31(22.26)$ & $16.72(11.45)$ \\
\hline & $\operatorname{CCSD}(\mathrm{T}, \mathrm{FULL}) / 6-311++\mathrm{G} * *$ & $9.73(9.96)$ & 24.95(19.90) & $15.21(9.94)$ \\
\hline \multirow{6}{*}{3} & $B 3 L Y P / 6-311++G^{* *}$ & $12.22(12.28)$ & $25.46(18.95)$ & $13.23(6.67)$ \\
\hline & B3LYP/6-311++G(3df,3pd) & $11.67(11.73)$ & $25.07(18.56)$ & $13.40(6.84)$ \\
\hline & B3LYP/AUG-cc-pVTZ & $11.67(11.73)$ & $25.03(18.52)$ & $13.36(6.79)$ \\
\hline & $M P 2(F U L L) / 6-311++G^{* *}$ & $10.47(10.82)$ & $27.54(20.46)$ & $17.07(9.64)$ \\
\hline & MP2(FULL)/6-311++G** & $10.72(10.78)$ & $27.57(21.07)$ & $16.85(10.29)$ \\
\hline & MP3(FULL)/6-311++G** & $9.90(9.95)$ & $32.22(25.72)$ & $22.33(15.76)$ \\
\hline
\end{tabular}

${ }^{a}$ The italics refer to the full geometry optimizations at those levels.

${ }^{b}$ The data in parentheses refer to the results considering ZPVE corrections at the B3LYP/6-311++G** level of theory except for those of full geometry optimizations at the MP2(FULL)/6-311++G** level of theory.

${ }^{c}$ The numbers of $0,1,2$, and 3 refer to those of the un-, mono-, di-, and trihydrated cases, respectively. 